\begin{tabular}{|c|c|c|}
\hline & Our Nature & $\begin{array}{l}\text { ISSN: 1991-2951 (Print) } \\
\text { ISSN: 2091-2781 (Online) }\end{array}$ \\
\hline $\begin{array}{l}\text { Noture Consenvation \& } \\
\text { Heallh Care Council } \\
\text { Blothoga Nepod }\end{array}$ & Journal homepage: http://nepjol.info/index.php/ON & (c) ${ }_{\mathrm{BY}}$ (b) \\
\hline
\end{tabular}

\title{
Effect of Bamboo Substrate on Growth and Production of Carp and Small Indigenous Fish
}

\author{
Rajan Poudel $^{1}$, Sunila Rai* ${ }^{1}$, Dilip K Jha ${ }^{1}$ and Ram Bhajan Mandal ${ }^{2}$ \\ ${ }^{1}$ Fisheries Program, Agriculture and Forestry University, Rampur, Chitwan, Nepal \\ ${ }^{2}$ Lamjung Campus, Institute of Agriculture and Animal Science, Sundar Bazar, Lamjung, Nepal \\ *Corresponding Email: sunilarai10@gmail.com
}

\begin{abstract}
A field trial was conducted to examine effects of substrates on yield of carp and SIS at Sukranagar Village, Chitwan district for 210 days in farmer's pond. The experiment was designed with four treatments: $\mathrm{T}_{1}$ (carp only), $\mathrm{T}_{2}$ (carp+SIS), $\mathrm{T}_{3}$ (carp+substrate) and $\mathrm{T}_{4}$ (carp+SIS+substrate) with four replicates. Ponds were stocked with fingerlings of six carp species at a rate of 20,000 fish/ha and two Small indigenous fish species at a rate of 50,000 fish/ha in 1:1 ratio. Prior to stocking of fish, split bamboo mats were fixed in substrate ponds covering $2 \%$ of pond surface area to enhance periphyton growth. Carp were fed with dough of rice bran and mustard oil cake $(1: 1)$ at the rate of $3 \% \mathrm{BW}$ daily while Grass carp was fed with grass and banana leaves at $50 \%$ BW. Growth and yield of Rohu and Common carp, combined net fish yield and gross margin were found higher $(\mathrm{p}<0.05)$ in carp ponds with substrate.
\end{abstract}

Key words: Esomus danricus, Puntius sophore, periphyton, net fish yield

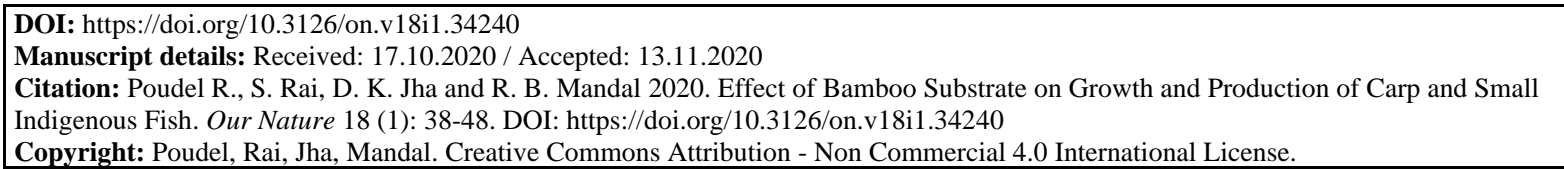

\section{Introduction}

Carp polyculture is the major aquaculture system in Nepal whereas carp-SIS polyculture is a nutrition sensitive aquaculture system suitable for rural farmers (CFPCC, 2019; Rai et al., 2012). Semi-intensive carp polyculture is the common practice where the feed cost is up to 70 percent of total operational cost (Yadav and Rai, 2014) which is expensive for small scale farmers. Thus it is essential to reduce feed cost to increase optimum profit. Adding substrates in ponds is found to increase carp growth and production, and reduce feed cost by providing harvestable natural food periphyton (Jha et al., 2018; Rai et al., 2019). Carp such as Rohu (Labeo rohita), Catla (Catla catla) and Common carp (Cyprinus carpio) are periphyton feeder (Rai et al., 2012) and are benefitted from putting substrate into the ponds. Carp had $47 \%$ higher total weight gain in no feeding (Rai et al., 2007) and 55\% higher yield in half feeding (Rai et al., 2019) periphyton enhanced systems indicated its potential to reduce feed input and cost.

There are large numbers of small scale carp farmers who sell most of their fish from ponds for family income rather consumed. Moreover, carp contain low micro-nutrients to contribute less to support their family nutrition. In contrast SIS (Small Indigenous Fish Species) are rich in micro-nutrients such as vitamin A, calcium, iron, zinc, phosphorus much needed for child and women (Roos et al., 2007; Rai et al., 2014). SIS such as Mara/Dhawai (Amblypharyngodon mola), Dedhuwa (Esomus danricus) and Pothi (Puntius sophore) have been reported to contain high amount of vitamin-A, iron and calcium, respectively (Roos et al., 2006; Rai et al., 2014). 


\section{Our Nature | December 2020 | 18 (1): 38-48}

Food and Agriculture Organization (2017) has recognized SIS as an important component of nutrition-sensitive agri-food system, thus increased consumption of SIS can combat existing malnutrition problem in Nepal. In Nepal, about $41 \%$ of children less than 5 years of age are stunted (UNICEF, 2012) and 48\% are anemic (MoHP, 2006). SIS can be harvested intermittently to increase household consumption and are eaten whole without plate waste (Rai et al., 2012; Thilsted et al., 1997). Growing SIS along with carp in household ponds is an appropriate way of increasing SIS consumption and income generation among small scale farmers (Rai et al., 2012).

Carp polyculture in periphyton enhanced system has been studied substantially in Bangladesh (Azim et al., 2002, 2004) and India (Ramesh et al., 1999; Mridula et al., 2003, 2004; Gangadhar and Keshavnath, 2012; but very few studies on carp-SIS polyculture in periphyton enhanced system have been done (Jha et al., 2018; Rai et al., 2018; Rai et al., 2019). Among the substrates used for periphyton enhancement, bamboo has performed best (Azim et al., 2002; Rai et al., 2007; Jha et al., 2018; Rai et al., 2019) and its potential to use as a periphyton substrates is higher due to its wide availability in the village. Farmers can have multiple benefits from bamboo substrate because it protects pond fish from poaching, improves water quality and eases harvesting of local snail Ghungi (Bellamya bengalensis). Present study aims to assesses potential of carp (Silver carp Hypophthalmichthys molitrix, Bighead carp Aristichthys nobilis, Grass carp Ctenopharyngodon idella, Common carp, Rohu and Mrigal Cirrhinus mrigala) and SIS (Dedhuwa and Pothi) polyculture in periphyton enhanced system using split bamboo mat as a periphyton substrate with full feeding.

\section{Materials and methods \\ Experimental site and design}

The field trial was conducted for 210 days from 20 May to 28 December 2014 in sixteen ponds of farmers in Sukranagar Village Development Committee (VDC) of Chitwan district. Average size of pond was $121.8 \mathrm{~m}^{2}$ (range $90-160 \mathrm{~m}^{2}$ ). The experiment was conducted in a completely randomized design (CRD). The experiment included four treatments i) $T_{1}$ (carp only), ii) $T_{2}$ (carp+SIS), iii) $\mathrm{T}_{3}$ (carp+substrate) and iv) $\mathrm{T}_{4}$ (carp+SIS+substrate), each treatment with four replicates.

\section{Pond preparation}

Prior to stocking, all ponds were dried up and limed with agriculture lime $\left(\mathrm{CaCO}_{3}\right)$ at the rate of $500 \mathrm{~kg} / \mathrm{ha}$. After 15 days of liming, cow dung was applied at the rate of $36 \mathrm{t} / \mathrm{ha}$. Water was filled to a depth of $1.2 \mathrm{~m}$. After water filling urea and Diammonium Phosphate (DAP) were applied at the rate of $470 \mathrm{~g} / 100 \mathrm{~m}^{2} /$ week and 350 $\mathrm{g} / 100 \mathrm{~m}^{2} /$ week, respectively. Similar rates of urea and DAP were used fortnightly throughout the trial period.

\section{Substrate preparation and installation}

Bamboo substrate in the form of mat was installed in substrate treatments $\left(\mathrm{T}_{3}\right.$ carp+substrate and $\mathrm{T}_{4}$ carp+SIS+substrate) for periphyton growth. Whole bamboo was split into $3-5 \mathrm{~cm}$ broad slats each with an average length of $1 \mathrm{~m}$. These slats were then tied into a rectangular bamboo mat by using string. Sufficient space between slats was kept to allow fish browsed on attached periphyton. Bamboo mats were suspended vertically by fixing lower ends to the pond bottom. Bamboo mats covered an area equivalent to $2 \%$ of total pond surface area.

\section{Stocking of carp and SIS}

Carp fingerlings were obtained from the Fisheries Development Centre, Bhandara and private hatcheries in Chitwan while SIS were collected from local ponds and canals. Stocking of fish was done after 7 days of initial fertilization. All ponds were stocked with Silver carp (36.3 $\pm 0.8 \mathrm{~g})$, Bighead carp $(49.9 \pm 0.4 \mathrm{~g})$, Grass carp $(52.0 \pm 0.7 \mathrm{~g})$, Common carp $(35.4 \pm 0.8 \mathrm{~g})$, Rohu (33.7 $\pm 0.9 \mathrm{~g})$ and Mrigal $(21.4 \pm 0.2 \mathrm{~g})$ at densities of $4000,1000,6000$, 3000,4000 and 2000 fingerlings per hectare respectively. Similarly, Dedhuwa and Pothi were added at a density of 50,000 fish per hectare at a ratio of $1: 1$ to SIS treatment ponds: carp+SIS $\left(\mathrm{T}_{2}\right)$ and carp+SIS+substrate $\left(\mathrm{T}_{4}\right)$.

\section{Feed and feeding}

Carps were fed with freshly made dough of rice bran and mustard oil cake mixed at 1:1 ratio every morning $(10 \mathrm{am})$ at the rate of $3 \% \mathrm{BW}$. Feed was given in a bamboo tray fixed at one corner of each pond. Grass carp was fed daily morning with locally available grass, banana leaves and legume leaves at the rate of $50 \% \mathrm{BW}$. Feed rations were adjusted monthly based on sampled weight of carp. 


\section{Our Nature | December 2020 | 18 (1): 38-48}

\section{Water quality analysis}

Water temperature, dissolved oxygen (DO) and $\mathrm{pH}$ was recorded weekly at 7-8 am in situ using DO meter and $\mathrm{pH}$ meter, respectively while transparency was measured weekly at 9.00 am using Secchi disk.

\section{Fish growth check and harvesting}

About $10 \%$ population of each species of carp were sampled monthly and weighed to determine the growth. Final harvesting of all carps and SIS was done by draining each pond completely. On harvesting, carp were counted and their batch weight was recorded while SIS were weighed in bulk and total biomass was taken. Household consumption of SIS through partial harvesting and carp mortality was determined from the record book provided to farmers. Each farmer was provided with a note book to record SIS consumed and carp mortality during experimental period.

\section{Economic analysis}

Gross margin analysis was performed to determine the income and profit from each treatment (Shang, 1990). Gross margin for each treatment was determined by deducting total variable cost from gross income. Variable cost and gross income was estimated based on current local market prices. Price for fingerlings of carp was NRs. 5 per piece while that of SIS were NRs 0.10 per piece. Selling price of harvested carp was NRs. $250 / \mathrm{kg}$ while that of Dedhuwa and Pothi was NRs $200 / \mathrm{kg}$. Prices for urea, DAP and feed was NRs. 30,50 and $29 / \mathrm{kg}$, respectively.

\section{Statistical analysis}

Data of growth and yield of carps and SIS, water quality, gross margin among different treatments were analysed statistically using one-way analysis of variance (ANOVA) followed by Duncan's multiple range test (DMRT). Data of growth and yield of carp between carp polyculture with and without bamboo substrate and that of SIS between carp+SIS polyculture with and without bamboo substrate were analysed by using Student's t-test. Differences were considered significant at an alpha level of 0.05 $(\mathrm{P}<0.05)$. All means are given with \pm 1 standard error (SE).

\section{Results}

\section{Growth and yield of carps among treatments}

Species wise growth, survival and yields of carp in different treatments, GFY and NFY of carp, GFY and NFY of SIS and combined (carp+SIS) GFY and NFY, and FCR are shown in Table 1. Among carps Rohu, Silver carp, Bighead carp and Common carp showed variation $(p<0.05)$ in growth, yield and survival among treatments while Mrigal and Grass carp did not ( $p>0.05$ ). Growth, yields and survival of Rohu was significantly $(\mathrm{p}<0.05)$ highest in carp+substrate treatment intermediate in carp+SIS+substrate treatment and lower in carp only and carp+SIS treatments. Silver carp and Bighead carp being planktivore fish had significantly $(\mathrm{p}<0.05)$ higher growth, survival and yields in carp only treatment. Total harvest weight, total weight gain, survival, GFY and NFY of Silver carp was lower $(\mathrm{p}<0.05)$ in carp+SIS+substrate treatment but these values were same with carp+SIS and carp+substrate treatments. Similarly, total harvest weight and total weight gain, survival, GFY and NFY of Bighead carp were higher $(\mathrm{p}<0.05)$ in carp only treatment than carp+SIS treatment but were same with carp+substrate and carp+SIS+substrate treatments. Average harvesting weight, average weight gain and daily weight gain of Common carp was significantly $(p<0.05)$ higher in substrate treatments than nonsubstrate treatments while GFY and NFY was significantly $(\mathrm{p}<0.05)$ higher in carp+SIS, carp+substrate and carp+SIS+substrate treatments than in carp only treatment.

Table 1. Growth performance of carps in different treatments during experimental period (Mean $\pm \mathrm{SE})$

\begin{tabular}{lllll}
\hline \multicolumn{1}{c}{ Parameters } & \multicolumn{1}{c}{$\mathrm{T}_{1}$} & \multicolumn{1}{c}{$\mathrm{T}_{2}$} & \multicolumn{1}{c}{$\mathrm{T}_{3}$} & \multicolumn{1}{c}{$\mathrm{T}_{4}$} \\
\hline Rohu & & & & \\
Average stocking weight $(\mathrm{g} /$ fish $)$ & $34.1 \pm 0.3^{\mathrm{a}}$ & $34.3 \pm 1.5^{\mathrm{a}}$ & $35.4 \pm 1.4^{\mathrm{a}}$ & $31.3 \pm 2.7^{\mathrm{a}}$ \\
Total stocking weight $\left(\mathrm{kg} / 100 \mathrm{~m}^{2}\right)$ & $1.4 \pm 0.0^{\mathrm{a}}$ & $1.4 \pm 0.1^{\mathrm{a}}$ & $1.4 \pm 0.1^{\mathrm{a}}$ & $1.3 \pm 0.1^{\mathrm{a}}$ \\
Average harvesting weight $(\mathrm{g} / \mathrm{fish})$ & $171.8 \pm 2.9^{\mathrm{b}}$ & $174.1 \pm 1.7^{\mathrm{b}}$ & $192.8 \pm 0.6^{\mathrm{a}}$ & $189.5 \pm 1.7^{\mathrm{a}}$ \\
Total harvesting weight $\left(\mathrm{kg} / 100 \mathrm{~m}^{2}\right)$ & $4.8 \pm 0.2^{\mathrm{c}}$ & $4.6 \pm 0.2^{\mathrm{c}}$ & $6.5 \pm 0.2^{\mathrm{a}}$ & $5.4 \pm 0.2^{\mathrm{b}}$ \\
Average weight gain $(\mathrm{g} / \mathrm{fish})$ & $137.8 \pm 2.6^{\mathrm{a}}$ & $139.8 \pm 1.7^{\mathrm{a}}$ & $157.3 \pm 1.9^{\mathrm{a}}$ & $158.2 \pm 1.8^{\mathrm{a}}$ \\
Total weight gain $\left(\mathrm{kg} / 100 \mathrm{~m}^{2}\right)$ & $3.4 \pm 0.2^{\mathrm{c}}$ & $3.2 \pm 0.3^{\mathrm{c}}$ & $5.0 \pm 0.1^{\mathrm{a}}$ & $4.1 \pm 0.3^{\mathrm{b}}$ \\
Daily weight gain (g/fish/day) & $0.7 \pm 0.0^{\mathrm{b}}$ & $0.7 \pm 0.0^{\mathrm{b}}$ & $0.8 \pm 0.0^{\mathrm{a}}$ & $0.8 \pm 0.0^{\mathrm{a}}$ \\
Survival $(\%)$ & $69.1 \pm 1.4^{\mathrm{b}}$ & $65.5 \pm 3.2^{\mathrm{b}}$ & $83.3 \pm 2.4^{\mathrm{a}}$ & $71.3 \pm 3.3^{\mathrm{b}}$
\end{tabular}


Our Nature | December 2020 | 18 (1): 38-48

\begin{tabular}{|c|c|c|c|c|}
\hline Parameters & $\mathrm{T}_{1}$ & $\mathrm{~T}_{2}$ & $\mathrm{~T}_{3}$ & $\mathrm{~T}_{4}$ \\
\hline Extrapolated GFY (t/ha/yr) & $0.8 \pm 0.0^{\mathrm{c}}$ & $0.8 \pm 0.0^{\mathrm{c}}$ & $1.1 \pm 0.0^{\mathrm{a}}$ & $0.9 \pm 0.0^{\mathrm{b}}$ \\
\hline Extrapolated NFY (t/ha/yr) & $0.6 \pm 0.0^{\mathrm{c}}$ & $0.6 \pm 0.0^{\mathrm{c}}$ & $0.9 \pm 0.0^{\mathrm{a}}$ & $0.7 \pm 0.0^{\mathrm{b}}$ \\
\hline \multicolumn{5}{|l|}{ Mrigal } \\
\hline Average stocking weight (g/fish) & $21.3 \pm 0.4^{\mathrm{a}}$ & $22.1 \pm 0.4^{\mathrm{a}}$ & $21.4 \pm 0.8^{\mathrm{a}}$ & $20.9 \pm 0.7^{\mathrm{a}}$ \\
\hline Total stocking weight $\left(\mathrm{kg} / 100 \mathrm{~m}^{2}\right)$ & $0.4 \pm 0.0^{\mathrm{a}}$ & $0.4 \pm 0.0^{\mathrm{a}}$ & $0.4 \pm 0.0^{\mathrm{a}}$ & $0.4 \pm 0.0^{\mathrm{a}}$ \\
\hline Average harvesting weight (g/fish) & $167.4 \pm 6.2^{\mathrm{a}}$ & $171.8 \pm 3.0^{\mathrm{a}}$ & $175.5 \pm 2.1^{\mathrm{a}}$ & $171.2 \pm 3.0^{\mathrm{a}}$ \\
\hline Total harvesting weight $\left(\mathrm{kg} / 100 \mathrm{~m}^{2}\right)$ & $2.5 \pm 0.1^{\mathrm{a}}$ & $2.4 \pm 0.1^{\mathrm{a}}$ & $2.5 \pm 0.4^{\mathrm{a}}$ & $2.2 \pm 0.2^{\mathrm{a}}$ \\
\hline Average weight gain (g/fish) & $146.2 \pm 6.5^{\mathrm{a}}$ & $149.7 \pm 3.1^{\mathrm{a}}$ & $154.1 \pm 2.1^{\mathrm{a}}$ & $150.3 \pm 2.4^{\mathrm{a}}$ \\
\hline Total weight gain $\left(\mathrm{kg} / 100 \mathrm{~m}^{2}\right)$ & $2.0 \pm 0.1^{\mathrm{a}}$ & $1.9 \pm 0.1^{\mathrm{a}}$ & $2.1 \pm 0.4^{\mathrm{a}}$ & $1.8 \pm 0.2^{\mathrm{a}}$ \\
\hline Daily weight gain (g/fish/day) & $0.7 \pm 0.0^{\mathrm{a}}$ & $0.7 \pm 0.0^{\mathrm{a}}$ & $0.7 \pm 0.0^{\mathrm{a}}$ & $0.7 \pm 0.0^{\mathrm{a}}$ \\
\hline Survival (\%) & $72.7 \pm 2.7^{\mathrm{a}}$ & $67.8 \pm 2.6^{\mathrm{a}}$ & $71.4 \pm 10.9^{\mathrm{a}}$ & $63.8 \pm 5.2^{\mathrm{a}}$ \\
\hline Extrapolated GFY (t/ha/yr) & $0.4 \pm 0.0^{\mathrm{a}}$ & $0.4 \pm 0.0^{\mathrm{a}}$ & $0.4 \pm 0.1^{\mathrm{a}}$ & $0.4 \pm 0.0^{\mathrm{a}}$ \\
\hline Extrapolated NFY (t/ha/yr) & $0.4 \pm 0.0^{\mathrm{a}}$ & $0.3 \pm 0.0^{\mathrm{a}}$ & $0.4 \pm 0.1^{\mathrm{a}}$ & $0.3 \pm 0.0^{\mathrm{a}}$ \\
\hline \multicolumn{5}{|l|}{ Silver carp } \\
\hline Average stocking weight (g/fish) & $35.4 \pm 0.2^{\mathrm{a}}$ & $35.6 \pm 0.4^{\mathrm{a}}$ & $37.5 \pm 0.3^{\mathrm{a}}$ & $36.8 \pm 1.4^{\mathrm{a}}$ \\
\hline Total stocking weight $\left(\mathrm{kg} / 100 \mathrm{~m}^{2}\right)$ & $1.4 \pm 0.0^{\mathrm{a}}$ & $1.4 \pm 0.0^{\mathrm{a}}$ & $1.5 \pm 0.0^{\mathrm{a}}$ & $1.5 \pm 0.1^{\mathrm{a}}$ \\
\hline Average harvesting weight (g/fish) & $360.1 \pm 6.2^{\mathrm{a}}$ & $355.1 \pm 5.4^{\mathrm{a}}$ & $364.4 \pm 4.7^{\mathrm{a}}$ & $360.5 \pm 2.1^{\mathrm{a}}$ \\
\hline Total harvesting weight $\left(\mathrm{kg} / 100 \mathrm{~m}^{2}\right)$ & $8.2 \pm 0.7^{\mathrm{a}}$ & $7.2 \pm 0.8^{\mathrm{ab}}$ & $7.5 \pm 0.7^{\mathrm{ab}}$ & $5.9 \pm 0.1^{\mathrm{b}}$ \\
\hline Average weight gain (g/fish) & $324.8 \pm 6.2^{\mathrm{a}}$ & $319.5 \pm 5.6^{\mathrm{a}}$ & $326.8 \pm 4.8^{\mathrm{a}}$ & $323.7 \pm 1.7^{\mathrm{a}}$ \\
\hline Total weight gain $\left(\mathrm{kg} / 100 \mathrm{~m}^{2}\right)$ & $6.8 \pm 0.7^{\mathrm{a}}$ & $5.8 \pm 0.8^{\mathrm{ab}}$ & $6.0 \pm 0.7^{\mathrm{ab}}$ & $4.5 \pm 0.1^{\mathrm{b}}$ \\
\hline Daily weight gain (g/fish/day) & $1.5 \pm 0.0^{\mathrm{a}}$ & $1.5 \pm 0.0^{\mathrm{a}}$ & $1.6 \pm 0.0^{\mathrm{a}}$ & $1.5 \pm 0.0^{\mathrm{a}}$ \\
\hline Survival (\%) & $56.4 \pm 4.4^{\mathrm{a}}$ & $50.8 \pm 5.2^{\mathrm{ab}}$ & $51.5 \pm 4.7^{\mathrm{ab}}$ & $41.3 \pm 0.7^{b}$ \\
\hline Extrapolated GFY (t/ha/yr) & $1.4 \pm 0.1^{\mathrm{a}}$ & $1.3 \pm 0.1^{\mathrm{ab}}$ & $1.3 \pm 0.1^{\mathrm{ab}}$ & $1.0 \pm 0.0^{\mathrm{b}}$ \\
\hline Extrapolated NFY (t/ha/yr) & $1.2 \pm 0.1^{\mathrm{a}}$ & $1.0 \pm 0.1^{\mathrm{ab}}$ & $1.0 \pm 0.1^{\mathrm{ab}}$ & $0.8 \pm 0.0^{\mathrm{b}}$ \\
\hline \multicolumn{5}{|l|}{ Bighead carp } \\
\hline Average stocking weight (g/fish) & $51.1 \pm 0.4^{\mathrm{a}}$ & $49.0 \pm 1.6^{\mathrm{a}}$ & $50.0 \pm 1.4^{\mathrm{a}}$ & $49.3 \pm 1.7^{\mathrm{a}}$ \\
\hline Total stocking weight $\left(\mathrm{kg} / 100 \mathrm{~m}^{2}\right)$ & $0.5 \pm 0.0^{\mathrm{a}}$ & $0.5 \pm 0.0^{\mathrm{a}}$ & $0.5 \pm 0.0^{\mathrm{a}}$ & $0.5 \pm 0.0^{\mathrm{a}}$ \\
\hline Average harvesting weight (g/fish) & $451.5 \pm 16.6^{\mathrm{a}}$ & $446.1 \pm 29.4^{\mathrm{a}}$ & $463.5 \pm 12.7^{\mathrm{a}}$ & $461.5 \pm 15.3^{\mathrm{a}}$ \\
\hline Total harvesting weight $\left(\mathrm{kg} / 100 \mathrm{~m}^{2}\right)$ & $2.6 \pm 0.3^{\mathrm{a}}$ & $1.8 \pm 0.2^{\mathrm{b}}$ & $2.1 \pm 0.2^{\mathrm{ab}}$ & $2.1 \pm 0.1^{\mathrm{ab}}$ \\
\hline Average weight gain (g/fish) & $400.5 \pm 16.3^{\mathrm{a}}$ & $397.1 \pm 29.3^{\mathrm{a}}$ & $413.5 \pm 11.8^{\mathrm{a}}$ & $412.2 \pm 16.0^{\mathrm{a}}$ \\
\hline Total weight gain $\left(\mathrm{kg} / 100 \mathrm{~m}^{2}\right)$ & $2.0 \pm 0.3^{\mathrm{a}}$ & $1.3 \pm 0.2^{b}$ & $1.6 \pm 0.2^{\mathrm{ab}}$ & $1.6 \pm 0.1^{\mathrm{ab}}$ \\
\hline Daily weight gain (g/fish/day) & $1.9 \pm 0.1^{\mathrm{a}}$ & $1.9 \pm 0.1^{\mathrm{a}}$ & $2.0 \pm 0.1^{\mathrm{a}}$ & $2.0 \pm 0.1^{\mathrm{a}}$ \\
\hline Survival (\%) & $57.8 \pm 7.1^{\mathrm{a}}$ & $40.2 \pm 5.0^{\mathrm{b}}$ & $44.9 \pm 4.2^{\mathrm{ab}}$ & $45.0 \pm 2.9^{\mathrm{ab}}$ \\
\hline Extrapolated GFY (t/ha/yr) & $0.5 \pm 0.0^{\mathrm{a}}$ & $0.3 \pm 0.0^{\mathrm{b}}$ & $0.4 \pm 0.0^{\mathrm{ab}}$ & $0.4 \pm 0.0^{\mathrm{ab}}$ \\
\hline Extrapolated NFY (t/ha/yr) & $0.4 \pm 0.0^{\mathrm{a}}$ & $0.2 \pm 0.0^{\mathrm{b}}$ & $0.3 \pm 0.0^{\mathrm{ab}}$ & $0.3 \pm 0.0^{\mathrm{ab}}$ \\
\hline \multicolumn{5}{|l|}{ Grass carp } \\
\hline Average stocking weight (g/fish) & $51.1 \pm 0.7^{\mathrm{b}}$ & $51.4 \pm 0.9^{\mathrm{b}}$ & $51.5 \pm 0.6^{\mathrm{b}}$ & $54.0 \pm 0.8^{\mathrm{a}}$ \\
\hline Total stocking weight $\left(\mathrm{kg} / 100 \mathrm{~m}^{2}\right)$ & $3.1 \pm 0.0^{\mathrm{b}}$ & $3.1 \pm 0.1^{\mathrm{b}}$ & $3.1 \pm 0.0^{\mathrm{b}}$ & $3.2 \pm 0.0^{\mathrm{a}}$ \\
\hline Average harvesting weight (g/fish) & $377.6 \pm 7.1^{\mathrm{a}}$ & $357.5 \pm 10.1^{\mathrm{a}}$ & $392.1 \pm 22.1^{\mathrm{a}}$ & $375.0 \pm 7.7^{\mathrm{a}}$ \\
\hline Total harvesting weight $\left(\mathrm{kg} / 100 \mathrm{~m}^{2}\right)$ & $8.2 \pm 0.8^{\mathrm{a}}$ & $7.9 \pm 0.9^{\mathrm{a}}$ & $8.7 \pm 0.2^{\mathrm{a}}$ & $8.1 \pm 0.3^{\mathrm{a}}$ \\
\hline Average weight gain (g/fish) & $326.4 \pm 7.7^{\mathrm{a}}$ & $306.1 \pm 10.5^{\mathrm{a}}$ & $340.6 \pm 22.0^{\mathrm{a}}$ & $321.0 \pm 8.4^{\mathrm{a}}$ \\
\hline Total weight gain $\left(\mathrm{kg} / 100 \mathrm{~m}^{2}\right)$ & $5.8 \pm 0.9^{\mathrm{a}}$ & $4.8 \pm 0.7^{\mathrm{a}}$ & $5.6 \pm 0.3^{\mathrm{a}}$ & $4.8 \pm 0.3^{\mathrm{a}}$ \\
\hline Daily weight gain (g/fish/day) & $1.6 \pm 0.0^{\mathrm{a}}$ & $1.5 \pm 0.0^{\mathrm{a}}$ & $1.6 \pm 0.1^{\mathrm{a}}$ & $1.5 \pm 0.0^{\mathrm{a}}$ \\
\hline Survival $(\%)$ & $36.1 \pm 3.7^{\mathrm{a}}$ & $36.8 \pm 2.8^{\mathrm{a}}$ & $37.3 \pm 3.2^{\mathrm{a}}$ & $35.8 \pm 1.1^{\mathrm{a}}$ \\
\hline Extrapolated GFY (t/ha/yr) & $1.4 \pm 0.1^{\mathrm{a}}$ & $1.4 \pm 0.1^{\mathrm{a}}$ & $1.5 \pm 0.0^{\mathrm{a}}$ & $1.4 \pm 0.0^{\mathrm{a}}$ \\
\hline Extrapolated NFY (t/ha/yr) & $0.8 \pm 0.2^{\mathrm{a}}$ & $0.8 \pm 0.1^{\mathrm{a}}$ & $1.0 \pm 0.0^{\mathrm{a}}$ & $0.8 \pm 0.1^{\mathrm{a}}$ \\
\hline \multicolumn{5}{|l|}{ Common carp } \\
\hline Average stocking weight (g/fish) & $35.9 \pm 0.5^{\mathrm{a}}$ & $36.0 \pm 1.6^{\mathrm{a}}$ & $33.0 \pm 0.9^{\mathrm{a}}$ & $36.6 \pm 1.4^{\mathrm{a}}$ \\
\hline Total stocking weight $\left(\mathrm{kg} / 100 \mathrm{~m}^{2}\right)$ & $1.1 \pm 0.0^{\mathrm{a}}$ & $1.1 \pm 0.0^{\mathrm{a}}$ & $1.0 \pm 0.0^{\mathrm{a}}$ & $1.1 \pm 0.0^{\mathrm{a}}$ \\
\hline Average harvesting weight (g/fish) & $406.7 \pm 6.7^{\mathrm{b}}$ & $417.2 \pm 3.7^{\mathrm{b}}$ & $467.3 \pm 14.6^{\mathrm{a}}$ & $460.2 \pm 7.2^{\mathrm{a}}$ \\
\hline Total harvesting weight $\left(\mathrm{kg} / 100 \mathrm{~m}^{2}\right)$ & $4.9 \pm 0.2^{\mathrm{b}}$ & $5.7 \pm 0.3^{\mathrm{ab}}$ & $5.5 \pm 0.4^{\mathrm{ab}}$ & $6.0 \pm 0.3^{\mathrm{a}}$ \\
\hline Average weight gain (g/fish) & $370.8 \pm 7.0^{\mathrm{b}}$ & $381.1 \pm 2.4^{\mathrm{b}}$ & $434.4 \pm 14.8^{\mathrm{a}}$ & $423.7 \pm 7.2^{\mathrm{a}}$ \\
\hline Total weight gain $\left(\mathrm{kg} / 100 \mathrm{~m}^{2} /\right)$ & $3.8 \pm 0.2^{\mathrm{b}}$ & $4.7 \pm 0.3^{\mathrm{ab}}$ & $4.5 \pm 0.4^{\mathrm{ab}}$ & $4.9 \pm 0.3^{\mathrm{a}}$ \\
\hline Daily weight gain (g/fish/day) & $1.8 \pm 0.0^{\mathrm{b}}$ & $1.8 \pm 0.0^{\mathrm{b}}$ & $2.1 \pm 0.1^{\mathrm{a}}$ & $2.0 \pm 0.0^{\mathrm{a}}$ \\
\hline
\end{tabular}


Our Nature | December 2020 | 18 (1): 38-48

\begin{tabular}{lllll}
\hline \multicolumn{1}{c}{ Parameters } & \multicolumn{1}{c}{$\mathrm{T}_{1}$} & \multicolumn{1}{c}{$\mathrm{T}_{2}$} & $\mathrm{~T}_{3}$ & $\mathrm{~T}_{4}$ \\
\hline Survival (\%) & $40.0 \pm 1.6^{\mathrm{a}}$ & $43.8 \pm 2.3^{\mathrm{a}}$ & $39.1 \pm 2.5^{\mathrm{a}}$ & $43.3 \pm 2.4^{\mathrm{a}}$ \\
Extrapolated GFY (t/ha/yr) & $0.8 \pm 0.0^{\mathrm{b}}$ & $1.0 \pm 0.1^{\mathrm{a}}$ & $1.0 \pm 0.1^{\mathrm{a}}$ & $1.0 \pm 0.1^{\mathrm{a}}$ \\
Extrapolated NFY (t/ha/yr) & $0.7 \pm 0.0^{\mathrm{b}}$ & $0.8 \pm 0.1^{\mathrm{a}}$ & $0.8 \pm 0.1^{\mathrm{a}}$ & $0.8 \pm 0.0^{\mathrm{a}}$ \\
Combined & & & & \\
GFY of Carp only (t/ha/yr) & $5.40 \pm 0.06^{\mathrm{ab}}$ & $5.14 \pm 0.23^{\mathrm{b}}$ & $5.69 \pm 0.21^{\mathrm{a}}$ & $5.15 \pm 0.04^{\mathrm{b}}$ \\
NFY of Carp only (t/ha/yr) & $4.04 \pm 0.06^{\mathrm{ab}}$ & $3.77 \pm 0.24^{\mathrm{b}}$ & $4.31 \pm 0.13^{\mathrm{a}}$ & $3.77 \pm 0.01^{\mathrm{b}}$ \\
GFY of SIS only (t/ha/yr) & - & $0.28 \pm 0.02^{\mathrm{a}}$ & & $0.21 \pm 0.02^{\mathrm{a}}$ \\
NFY of SIS only (t/ha/yr) & - & $0.14 \pm 0.03^{\mathrm{a}}$ & & $0.06 \pm 0.02^{\mathrm{b}}$ \\
Combined GFY (t/ha/yr) & $5.40 \pm 0.06^{\mathrm{a}}$ & $5.41 \pm 0.24^{\mathrm{a}}$ & $5.69 \pm 0.21^{\mathrm{a}}$ & $5.36 \pm 0.05^{\mathrm{a}}$ \\
Combined NFY (t/ha/yr) & $4.04 \pm 0.06^{\mathrm{ab}}$ & $3.90 \pm 0.26^{\mathrm{ab}}$ & $4.31 \pm 0.13^{\mathrm{a}}$ & $3.82 \pm 0.03^{\mathrm{b}}$ \\
Feed Conversion Ratio & $3.34 \pm 0.07^{\mathrm{a}}$ & $3.39 \pm 0.12^{\mathrm{a}}$ & $3.23 \pm 0.02^{\mathrm{a}}$ & $3.33 \pm 0.06^{\mathrm{a}}$ \\
\hline
\end{tabular}

GFY=Gross Fish Yield, NFY=Net Fish Yield

Mean values with different superscript letters in the same row are significantly different $\mathrm{p}<0.05$.

GFY and NFY of carp was significantly higher $(p<0.05)$ in carp+substrate treatment than SIS included treatments but these values did not differ ( $p>0.05)$ with carp only treatment. Between SIS treatments, NFY of SIS was significantly $(\mathrm{p}<0.05)$ higher in without substrate treatment over substrate treatment. While combining yield of carp and SIS, it was found that combined NFY was significantly $(\mathrm{p}<0.05)$ higher in carp+substrate treatment than carp+SIS +substrate treatment but it did not differ with carp only treatment and carp+SIS treatment. Feed conversion ratio did not differ $(p>0.05)$ among treatments due to full feeding system.

\section{Growth and yield of carps in ponds with and without bamboo substrate}

Growth, yield and survival of different carp species in carp polyculture with and without substrate are shown in Table 2. Among carps growth and yield of Rohu and Common carp was found enhanced by bamboo substrate as these carp had higher growth and yield in ponds with substrate than without substrate. Average harvesting weight, total harvesting weight, average weight gain, total weight gain, GFY and NFY of Rohu was significantly higher $(p<0.05)$ in substrate ponds than without substrate ponds. Similarly, Common carp had significantly $(\mathrm{p}<0.05)$ higher average harvesting weight, average weight gain and daily weight gain in substrate added ponds than without substrate ponds.

Table 2. Growth performance of carps in treatments with $\left(T_{1}\right)$ and without $\left(T_{2}\right)$ bamboo substrate during experimental period (Mean \pm SE)

\begin{tabular}{lll}
\multicolumn{1}{c}{ Parameters } & \multicolumn{1}{c}{$\mathrm{T}_{1}$} & \multicolumn{1}{c}{$\mathrm{T}_{2}$} \\
\hline Rohu & & \\
Average stocking weight (g/fish) & $34.1 \pm 0.3^{\mathrm{a}}$ & $35.4 \pm 1.4^{\mathrm{a}}$ \\
Total stocking weight $\left(\mathrm{kg} / 100 \mathrm{~m}^{2}\right)$ & $1.4 \pm 0.0^{\mathrm{a}}$ & $1.4 \pm 0.1^{\mathrm{a}}$ \\
Average harvesting weight $(\mathrm{g} / \mathrm{fish})$ & $171.8 \pm 2.9^{\mathrm{b}}$ & $192.8 \pm 0.6^{\mathrm{a}}$ \\
Total harvesting weight $\left(\mathrm{kg} / 100 \mathrm{~m}^{2}\right)$ & $4.8 \pm 0.2^{\mathrm{b}}$ & $6.5 \pm 0.2^{\mathrm{a}}$ \\
Average weight gain $(\mathrm{g} / \mathrm{fish})$ & $137.8 \pm 2.6^{\mathrm{b}}$ & $157.3 \pm 1.9^{\mathrm{a}}$ \\
Total weight gain $\left(\mathrm{kg} / 100 \mathrm{~m}^{2}\right)$ & $3.4 \pm 0.2^{\mathrm{b}}$ & $5.0 \pm 0.1^{\mathrm{a}}$ \\
Daily weight gain (g/fish/day) & $0.7 \pm 0.0^{\mathrm{a}}$ & $0.7 \pm 0.0^{\mathrm{a}}$ \\
Survival $(\%)$ & $69.1 \pm 1.4^{\mathrm{b}}$ & $83.3 \pm 2.4^{\mathrm{a}}$ \\
Extrapolated GFY (t/ha/yr) & $0.8 \pm 0.0^{\mathrm{b}}$ & $1.1 \pm 0.0^{\mathrm{a}}$ \\
Extrapolated NFY (t/ha/yr) & $0.6 \pm 0.0^{\mathrm{b}}$ & $0.9 \pm 0.0^{\mathrm{a}}$ \\
Mrigal & & \\
Average stocking weight $(\mathrm{g} / \mathrm{fish})$ & $21.3 \pm 0.4^{\mathrm{a}}$ & $21.4 \pm 0.8^{\mathrm{a}}$ \\
Total stocking weight $\left(\mathrm{kg} / 100 \mathrm{~m}^{2}\right)$ & $0.4 \pm 0.0^{\mathrm{a}}$ & $0.4 \pm 0.0^{\mathrm{a}}$ \\
Average harvesting weight $(\mathrm{g} / \mathrm{fish})$ & $167.4 \pm 6.2^{\mathrm{a}}$ & $175.5 \pm 2.1^{\mathrm{a}}$ \\
Total harvesting weight $\left(\mathrm{kg} / 100 \mathrm{~m}^{2}\right)$ & $2.5 \pm 0.1^{\mathrm{a}}$ & $2.5 \pm 0.4^{\mathrm{a}}$ \\
Average weight gain $(\mathrm{g} / \mathrm{fish})$ & $146.2 \pm 6.5^{\mathrm{a}}$ & $154.1 \pm 2.1^{\mathrm{a}}$ \\
Total weight gain $\left(\mathrm{kg} / 100 \mathrm{~m}{ }^{2}\right)$ & $2.0 \pm 0.1^{\mathrm{a}}$ & $2.1 \pm 0.4^{\mathrm{a}}$
\end{tabular}


Our Nature | December 2020 | 18 (1): 38-48

\begin{tabular}{|c|c|c|}
\hline Parameters & $\mathrm{T}_{1}$ & $\mathrm{~T}_{2}$ \\
\hline Daily weight gain (g/fish/day) & $0.7 \pm 0.0^{\mathrm{a}}$ & $0.7 \pm 0.0^{\mathrm{a}}$ \\
\hline Survival $(\%)$ & $72.7 \pm 2.7^{\mathrm{a}}$ & $71.4 \pm 10.9^{\mathrm{a}}$ \\
\hline Extrapolated GFY (t/ha/yr) & $0.4 \pm 0.0^{\mathrm{a}}$ & $0.4 \pm 0.1^{\mathrm{a}}$ \\
\hline Extrapolated NFY (t/ha/yr) & $0.4 \pm 0.0^{\mathrm{a}}$ & $0.4 \pm 0.1^{\mathrm{a}}$ \\
\hline \multicolumn{3}{|l|}{ Silver carp } \\
\hline Average stocking weight (g/fish) & $35.4 \pm 0.2^{\mathrm{a}}$ & $37.5 \pm 0.3^{\mathrm{a}}$ \\
\hline Total stocking weight $\left(\mathrm{kg} / 100 \mathrm{~m}^{2}\right)$ & $1.4 \pm 0.0^{\mathrm{a}}$ & $1.5 \pm 0.0^{\mathrm{a}}$ \\
\hline Average harvesting weight (g/fish) & $360.1 \pm 6.2^{\mathrm{a}}$ & $364.4 \pm 4.7^{\mathrm{a}}$ \\
\hline Total harvesting weight $\left(\mathrm{kg} / 100 \mathrm{~m}^{2}\right)$ & $8.2 \pm 0.7^{\mathrm{a}}$ & $7.5 \pm 0.7^{\mathrm{a}}$ \\
\hline Average weight gain (g/fish) & $324.8 \pm 6.2^{\mathrm{a}}$ & $326.8 \pm 4.8^{\mathrm{a}}$ \\
\hline Total weight gain $\left(\mathrm{kg} / 100 \mathrm{~m}^{2}\right)$ & $6.8 \pm 0.7^{\mathrm{a}}$ & $6.0 \pm 0.7^{\mathrm{a}}$ \\
\hline Daily weight gain (g/fish/day) & $1.5 \pm 0.0^{\mathrm{a}}$ & $1.6 \pm 0.0^{\mathrm{a}}$ \\
\hline Survival $(\%)$ & $56.4 \pm 4.4^{\mathrm{a}}$ & $51.5 \pm 4.7^{\mathrm{a}}$ \\
\hline Extrapolated GFY (t/ha/yr) & $1.4 \pm 0.1^{\mathrm{a}}$ & $1.3 \pm 0.1^{\mathrm{a}}$ \\
\hline Extrapolated NFY (t/ha/yr) & $1.2 \pm 0.1^{\mathrm{a}}$ & $1.0 \pm 0.1^{\mathrm{a}}$ \\
\hline \multicolumn{3}{|l|}{ Bighead carp } \\
\hline Average stocking weight (g/fish) & $51.1 \pm 0.4^{\mathrm{a}}$ & $50.0 \pm 1.4^{\mathrm{a}}$ \\
\hline Total stocking weight $\left(\mathrm{kg} / 100 \mathrm{~m}^{2}\right)$ & $0.5 \pm 0.0^{\mathrm{a}}$ & $0.5 \pm 0.0^{\mathrm{a}}$ \\
\hline Average harvesting weight (g/fish) & $451.5 \pm 16.6^{\mathrm{a}}$ & $463.5 \pm 12.7^{\mathrm{a}}$ \\
\hline Total harvesting weight $\left(\mathrm{kg} / 100 \mathrm{~m}^{2}\right)$ & $2.6 \pm 0.3^{\mathrm{a}}$ & $2.1 \pm 0.2^{\mathrm{a}}$ \\
\hline Average weight gain (g/fish) & $400.5 \pm 16.3^{\mathrm{a}}$ & $413.5 \pm 11.8^{\mathrm{a}}$ \\
\hline Total weight gain $\left(\mathrm{kg} / 100 \mathrm{~m}^{2}\right)$ & $2.0 \pm 0.3^{\mathrm{a}}$ & $1.6 \pm 0.2^{\mathrm{a}}$ \\
\hline Daily weight gain (g/fish/day) & $1.9 \pm 0.1^{\mathrm{a}}$ & $2.0 \pm 0.1^{\mathrm{a}}$ \\
\hline Survival $(\%)$ & $57.8 \pm 7.1^{\mathrm{a}}$ & $44.9 \pm 4.2^{\mathrm{a}}$ \\
\hline Extrapolated GFY (t/ha/yr) & $0.4 \pm 0.0^{\mathrm{a}}$ & $0.4 \pm 0.0^{\mathrm{a}}$ \\
\hline Extrapolated NFY (t/ha/yr) & $0.4 \pm 0.0^{\mathrm{a}}$ & $0.3 \pm 0.0^{\mathrm{a}}$ \\
\hline \multicolumn{3}{|l|}{ Grass carp } \\
\hline Average stocking weight (g/fish) & $51.1 \pm 0.7^{\mathrm{a}}$ & $51.5 \pm 0.6^{\mathrm{a}}$ \\
\hline Total stocking weight $\left(\mathrm{kg} / 100 \mathrm{~m}^{2}\right)$ & $3.1 \pm 0.0^{\mathrm{a}}$ & $3.1 \pm 0.0^{\mathrm{a}}$ \\
\hline Average harvesting weight (g/fish) & $377.6 \pm 7.1^{\mathrm{a}}$ & $392.1 \pm 22.1^{\mathrm{a}}$ \\
\hline Total harvesting weight $\left(\mathrm{kg} / 100 \mathrm{~m}^{2}\right)$ & $8.2 \pm 0.8^{\mathrm{a}}$ & $8.7 \pm 0.2^{\mathrm{a}}$ \\
\hline Average weight gain (g/fish) & $326.4 \pm 7.7^{\mathrm{a}}$ & $340.6 \pm 22.0^{\mathrm{a}}$ \\
\hline Total weight gain $\left(\mathrm{kg} / 100 \mathrm{~m}^{2}\right)$ & $5.8 \pm 0.9^{\mathrm{a}}$ & $5.6 \pm 0.3^{\mathrm{a}}$ \\
\hline Daily weight gain (g/fish/day) & $1.6 \pm 0.0^{\mathrm{a}}$ & $1.6 \pm 0.1^{\mathrm{a}}$ \\
\hline Survival $(\%)$ & $36.1 \pm 3.7^{\mathrm{a}}$ & $37.3 \pm 3.2^{\mathrm{a}}$ \\
\hline Extrapolated GFY (t/ha/yr) & $1.4 \pm 0.1^{\mathrm{a}}$ & $1.5 \pm 0.0^{\mathrm{a}}$ \\
\hline Extrapolated NFY (t/ha/yr) & $0.9 \pm 0.2^{\mathrm{a}}$ & $1.0 \pm 0.0^{\mathrm{a}}$ \\
\hline \multicolumn{3}{|l|}{ Common carp } \\
\hline Average stocking weight (g/fish) & $35.9 \pm 0.5^{\mathrm{a}}$ & $33.0 \pm 0.9^{\mathrm{a}}$ \\
\hline Total stocking weight $\left(\mathrm{kg} / 100 \mathrm{~m}^{2}\right)$ & $1.1 \pm 0.0^{\mathrm{a}}$ & $1.0 \pm 0.0^{\mathrm{a}}$ \\
\hline Average harvesting weight (g/fish) & $406.7 \pm 6.7^{\mathrm{b}}$ & $467.3 \pm 14.6^{\mathrm{a}}$ \\
\hline Total harvesting weight $\left(\mathrm{kg} / 100 \mathrm{~m}^{2}\right)$ & $4.9 \pm 0.2^{\mathrm{a}}$ & $5.5 \pm 0.4^{\mathrm{a}}$ \\
\hline Average weight gain (g/fish) & $370.8 \pm 7.0^{\mathrm{b}}$ & $434.4 \pm 14.8^{\mathrm{a}}$ \\
\hline Total weight gain $\left(\mathrm{kg} / 100 \mathrm{~m}^{2}\right)$ & $3.8 \pm 0.2^{\mathrm{a}}$ & $4.5 \pm 0.4^{\mathrm{a}}$ \\
\hline Daily weight gain (g/fish/day) & $1.8 \pm 0.0^{\mathrm{b}}$ & $2.1 \pm 0.1^{\mathrm{a}}$ \\
\hline Survival $(\%)$ & $40.0 \pm 1.6^{\mathrm{a}}$ & $39.1 \pm 2.5^{\mathrm{a}}$ \\
\hline Extrapolated GFY (t/ha/yr) & $0.8 \pm 0.0^{\mathrm{a}}$ & $1.0 \pm 0.1^{\mathrm{a}}$ \\
\hline Extrapolated NFY (t/ha/yr) & $0.7 \pm 0.0^{\mathrm{a}}$ & $0.8 \pm 0.1^{\mathrm{a}}$ \\
\hline
\end{tabular}

GFY=Gross Fish Yield, NFY=Net Fish Yield

Mean values with different superscript letters in the same row are significantly different $\mathrm{p}<0.05$.

Yield of SIS in ponds with and without substrate

Yield of SIS is shown in Table 3. Total harvesting weight and NFY of SIS (Dedhuwa+Pothi) were significantly higher $(\mathrm{p}<0.05)$ in carp+SIS ponds without substrate than with substrate. However, total stocking weight and extrapolated GFY did not differ ( $>0.05)$ between two treatments. 
Table 3. Production of SIS (Dedhuwa+Pothi) in different treatments during experimental period $($ Mean \pm SE)

\begin{tabular}{lcccc}
\hline \multirow{2}{*}{ Parameters } & \multicolumn{4}{c}{ Treatments } \\
\cline { 2 - 5 } & $\mathrm{T}_{1}$ & \multicolumn{1}{c}{$\mathrm{T}_{2}$} & $\mathrm{~T}_{3}$ & $\mathrm{~T}_{4}$ \\
\hline Total Stocking Weight $\left(\mathrm{g} / 100 \mathrm{~m}^{2}\right)$ & - & $814.4 \pm 39.7^{\mathrm{a}}$ & - & $894.4 \pm 43.1^{\mathrm{a}}$ \\
Total Harvesting Weight $\left(\mathrm{g} / 100 \mathrm{~m}^{2}\right)$ & - & $1592.8 \pm 121.6^{\mathrm{a}}$ & - & $1228.8 \pm 87.2^{\mathrm{b}}$ \\
Extrapolated GFY $(\mathrm{t} / \mathrm{ha} / \mathrm{yr})$ & - & $0.28 \pm 0.02^{\mathrm{a}}$ & - & $0.21 \pm 0.02^{\mathrm{a}}$ \\
Extrapolated NFY $(\mathrm{t} / \mathrm{ha} / \mathrm{yr})$ & - & $0.14 \pm 0.03^{\mathrm{a}}$ & - & $0.06 \pm 0.02^{\mathrm{b}}$ \\
\hline
\end{tabular}

GFY=Gross Fish Yield, NFY=Net Fish Yield

Mean values with different superscript letters in the same row are significantly different $(p<0.05)$.

\section{Water quality parameters}

Water quality parameters in different treatments are shown in Table 4 . Water quality parameters were not critical and did not differ $(p>0.05)$ among treatments.

Table 4. Water quality parameters in different treatments during experimental period (Mean $\pm \mathrm{SE}$ )

\begin{tabular}{lllll}
\hline Parameters & $\mathrm{T}_{1}$ & $\mathrm{~T}_{2}$ & $\mathrm{~T}_{3}$ & \multicolumn{1}{c}{$\mathrm{T}_{4}$} \\
\hline $\mathrm{pH}$ & 7.5 & 7.3 & 7.2 & 7.2 \\
& $(6.9-8.0)$ & $(7.0-8.1)$ & $(6.9-8.1)$ & $(7.0-7.8)$ \\
DO & $4.8 \pm 0.2^{\mathrm{a}}$ & $4.7 \pm 0.2^{\mathrm{a}}$ & $5.2 \pm 0.2^{\mathrm{a}}$ & $4.7 \pm 0.2^{\mathrm{a}}$ \\
& $(2.1-7.0)$ & $(2.0-7.0)$ & $(2.4-6.9)$ & $(2.5-6.9)$ \\
Temperature & $29.6 \pm 0.3^{\mathrm{a}}$ & $29.3 \pm 0.2^{\mathrm{a}}$ & $29.5 \pm 0.7^{\mathrm{a}}$ & $28.7 \pm 0.2^{\mathrm{a}}$ \\
& $(28.0-31.5)$ & $(27.7-32.3)$ & $(27.0-32.3)$ & $(27.0-32.3)$ \\
Transparency & $26 \pm 1^{\mathrm{a}}$ & $25 \pm 1^{\mathrm{a}}$ & $26 \pm 0^{\mathrm{a}}$ & $24 \pm 0^{\mathrm{a}}$ \\
& $(16-40)$ & $(18-38)$ & $(17-36)$ & $(17-36)$ \\
\hline
\end{tabular}

Mean values with different superscript letters in the same row are significantly different $(\mathrm{p}<0.05)$. Values in parenthesis shows the range.

\section{Gross margin analysis}

Gross margin analysis of different treatments is shown in Table 5. Feed cost, variable cost and return from carp were significantly higher $(\mathrm{p}<0.05)$ in carp+substrate treatment corresponding to higher carp yield. Feed cost was higher in carp+substrate treatment than in carp+SIS+substrate treatment while it did not differ with carp only and carp+SIS treatment. Total variable cost in carp+substrate treatment was significantly higher $(\mathrm{p}<0.05)$ than in carp only and carp+SIS treatments while it did not show significant difference $(p>0.05)$ with
carp+SIS+substrate treatment. Carp ponds with substrate gave significantly higher $(\mathrm{p}<0.05)$ gross return from carp than in carp+SIS ponds and carp+SIS+substrate ponds while return in carp only ponds showed no significant difference ( $>0.05)$ with any other treatments. There was no significant difference $(p>0.05)$ in gross return from SIS among two treatments due to low and similar production. Similarly, combined gross return and gross margin showed no significant difference $(\mathrm{p}>0.05)$ among all treatments. 
Our Nature | December 2020 | 18 (1): 38-48

Table 5. Gross margin analysis of different treatments (NRs/100 $\left.\mathrm{m}^{2}\right)$ during 210 days

\begin{tabular}{lcccc}
\hline \multirow{2}{*}{ Variables } & \multicolumn{4}{c}{ Treatment } \\
\cline { 2 - 5 } & $\mathrm{T}_{1}$ & $\mathrm{~T}_{2}$ & $\mathrm{~T}_{3}$ & $\mathrm{~T}_{4}$ \\
\hline Carp Seed & 1000 & 1000 & 1000 & 1000 \\
SIS Seed & & 50 & & 50 \\
Fertilizer & 142 & 142 & 142 & 142 \\
Lime & 55 & 55 & 55 & 55 \\
Bamboo & & 125 & 125 \\
Feed & $3009 \pm 38^{\mathrm{ab}}$ & $2893 \pm 58^{\mathrm{ab}}$ & $3045 \pm 59^{\mathrm{a}}$ & $2864 \pm 36^{\mathrm{b}}$ \\
Total Variable cost & $4206 \pm 38^{\mathrm{b}}$ & $4140 \pm 58^{\mathrm{b}}$ & $4367 \pm 59^{\mathrm{a}}$ & $4236 \pm 36^{\mathrm{ab}}$ \\
Return & & & & \\
Carp & $7771 \pm 85^{\mathrm{ab}}$ & $7388 \pm 332^{\mathrm{b}}$ & $8181 \pm 169^{\mathrm{a}}$ & $7408 \pm 57^{\mathrm{b}}$ \\
SIS & & $163 \pm 8^{\mathrm{a}}$ & & $179 \pm 9^{\mathrm{a}}$ \\
Gross Return & $7771 \pm 85^{\mathrm{a}}$ & $7551 \pm 326^{\mathrm{a}}$ & $8181 \pm 169^{\mathrm{a}}$ & $7587 \pm 62^{\mathrm{a}}$ \\
Gross Margin & $3565 \pm 109^{\mathrm{a}}$ & $3411 \pm 291^{\mathrm{a}}$ & $3814 \pm 120^{\mathrm{a}}$ & $3351 \pm 87^{\mathrm{a}}$ \\
\hline
\end{tabular}

Mean values with different superscript letters in the same row are significantly different $(\mathrm{p}<0.05)$.

\section{Discussion}

Present study was carried out with 4 treatments: carp only $\left(\mathrm{T}_{1}\right)$, carp+SIS $\left(\mathrm{T}_{2}\right)$, carp+substrate $\left(\mathrm{T}_{3}\right)$ and carp+SIS+substrate $\left(\mathrm{T}_{4}\right)$ to identify appropriate production system based on total fish production, water quality and economic return, and to determine effects of substrate on growth and production of carp and SIS. Among carps Rohu and Common carp showed positive relation with split bamboo substrate and SIS. Rohu and Common carp had higher growth and yield in substrate treatments because both of them are periphyton feeder and obtained extra benefit from periphyton enhancement (Rai et al., 2007; Rai et al., 2012; Jha et al., 2018). Previous studies have shown that production of Rohu is about $70 \%$ higher in substrate added ponds compared to control (Wahab et al., 1999; Azim et al., 2001a and 2002a). In present experiment, there was an increase of $12.5-25 \%$ in GFY and $16.6-30 \%$ in NFY with added substrate. Azim et al. (2001b) also showed that an increase of $60 \%$ in yield of Rohu could be obtained with added periphyton substrate in carp polyculture. Higher $(\mathrm{p}<0.05)$ growth and yield of Common carp in substrate treatments indicated that periphyton colonized on the split bamboo mat benefitted Common carp. Common carp is a bottom feeder, thus there is probably no intense overlapping with surface feeder Dedhuwa and Pothi. Since experiment used full feeding, Common carp is benefitted by utilizing both supplementary feed and periphyton efficiently.

Silver carp and Bighead carp had higher yield and survival in carp ponds without substrate because both species are exclusively planktivore and filter feeder. Perhaps they could not utilize periphyton on the substrate (Rai et al., 2012; Jha et al., 2018). Significantly lower yield and survival of Silver carp in carp+SIS+substrate treatment than carp only treatment indicated niche overlapping between Silver carp and Dedhuwa and Pothi due to their surface feeding habit. Similarly significantly lower yield and survival of Bighead carp in carp+SIS treatment than carp only treatment indicated niche overlapping between Bighead carp and Dedhuwa and Pothi. Pothi primarily feeds on planktons and Dedhuwa secondarily feeds on planktons (Shrestha, 2008) and thus they may compete with planktivorous species and might have reduced growth and production of Silver carp and Bighead carp in treatments with SIS. Moreover, both carp species and Dedhuwa and Pothi utilize same surface niche which might have resulted space and food competition. Present result is against of carp production is not affected by SIS as reported by Gupta and Rai (2011) and Roos et al. (2006). Growth and production parameters of Mrigal and Grass carp showed no significant difference $(p>0.05)$ among different treatments.

Gross and net fish yields of carp in carp+substrate treatment was significantly higher $(p<0.05)$ than in carp+SIS treatment and carp+SIS+substrate treatment while values in carp only treatment showed no significant difference $(p>0.05)$ than any other treatments. This can be attributed to higher production of Rohu and Common carp in substrate treatments and lower yield of Silver carp and Bighead carp 


\section{Our Nature | December 2020 | 18 (1): 38-48}

in carp+SIS treatments. Combined NFY in carp+substrate treatment was significantly higher $(p<0.05)$ than in carp+SIS+substrate with intermediate values in carp only and carp+SIS treatments. This can be attributed to the higher yield of carp in carp+substrate treatment and yield of SIS which is added up in combined fish yield in carp+SIS treatment. Feed conversion ratio in all treatments showed no significant difference $(p>0.05)$ because full feeding was used in all treatments. This indicates that added periphyton substrate can be beneficial for periphytophagous species.

Growth and production parameters of Rohu in treatments with bamboo substrate were significantly higher $(\mathrm{p}<0.05)$ than in treatments without bamboo substrate. This effect can be attributed to the browsing habit of Rohu with fringed inferior lips. Rai and Yi (2012) found that Rohu and Catla nibbles most on kanchi (bamboo side branches) and even at a low substrate density in fed ponds Rohu gives better yield. An average increase of $125 \%$ in extrapolated GFY and $133 \%$ in extrapolated NFY of Rohu was found in treatments with substrate compared to treatments without substrate. The average harvesting weight, average weight gain and daily weight gain of Common carp was significantly higher $(\mathrm{p}<0.05)$ in treatments with substrate than treatments without substrate. This effect also can be attributed to periphytophagous behavior of Common carp (Keshavanath et al., 2001b; Saikia and Das, 2009; Rai et al., 2012). There was no significant effect of substrate on growth or production parameters of Mrigal, Silver carp, Bighead carp and Grass carp as these carps do not feed on periphyton.

Gross fish yield of SIS did not show significant difference $(p>0.05)$ in SIS treatments with and without substrate. However, total harvesting weight and NFY of SIS was significantly higher $(\mathrm{p}<0.05)$ in carp+SIS treatment without substrate than in substrate treatment which indicated that SIS did not utilize periphyton and have negative effect of bamboo substrate (Jha et al., 2018). Mean water quality parameters of all four treatments were within the desired range for carp polyculture. Bamboo substrate did not deteriorate the water quality

Cost for feed was significantly higher $(p<0.05)$ in carp+substrate treatment than in carp+SIS+substrate treatment while cost for feed in carp only treatment and carp+SIS treatment showed no significant difference than any other treatment $(p>0.05)$. This might be due to full feeding system used in the trial and adjusting quantity of feed based on monthly growth check of carp. Carps grew better in carp+substrate treatment which resulted into higher feed supply to the treatment. Similarly, total variable cost in carp+substrate treatment was significantly higher $(\mathrm{p}<0.05)$ than in carp only and carp+SIS treatments while the value did not differ ( $>0.05)$ with carp+SIS+substrate treatment. This may be due to added cost of bamboo for substrate as well as higher feed cost. As production of carp in carp+substrate treatment was significantly higher $(\mathrm{p}<0.05)$, return from fish was also higher compared to other treatments. Adding substrate did not reduce the feed cost because experiment was carried out in full feeding system. This result indicates that bamboo substrates added to ponds in full feeding system is profitable but it could not reduce the feed cost. So, feeding rate should be reduced in ponds using bamboo substrates to reduce feed cost.

Based on growth and production of carp and gross margin, carp polyculture using bamboo substrate is better. The technology is environmentally friendly and viable so it should be promoted for small scale farmers in Nepal and South Asia where carps are grown widely.

\section{Acknowledgements}

Authors would like to thank Mr. Sovan Mahato and Ms. Usha Chaudhary for their help to conduct the field trial. We are also thankful to all farmers who participated in the trial. We are grateful to Assistant Professor Rahul Ranjan for helping in data analysis. This research was a part of Finnish project supported by AgriCord.

\section{References}

Azim, M. E., M. A. Wahab, A. A. Van Dam, M. C. M. Beveridge, A. Milstein, and M. C. J. Verdegem 2001a. Optimization of fertilization rate for maximizing periphyton production on artificial substrates and the implications for periphyton based aquaculture. Aquaculture Research 32: 749-760.

Azim, M. E., M. A. Wahab, A. A. Van Dam, M. C. M. Beveridge, E. A. Huisman, and M. C. J. Verdegem 2001b. Optimization of stocking ratios of two Indian major carps, rohu (Labeo rohita Ham.) and catla (Catla catla Ham.) in a periphyton-based aquaculture system." Aquaculture 203: 33-49. 


\section{Our Nature | December 2020 | 18 (1): 38-48}

Azim, M. E., M. A. Wahab, P. K. Biswas, T. Asaeda, T. Fujino, and M. C. J. Verdegem 2004. The effect of periphyton substrate density on production in freshwater polyculture ponds." Aquaculture 232: 441-453.

Azim, M. E., M. C. J. Verdegem, H. Khatoon, M. A. Wahab, A. A. Van Dam, and M. C. M. Beveridge 2002b. A comparison of fertilization, feeding and three periphyton substrates for increasing fish production in freshwater pond aquaculture in Bangladesh." Aquaculture 212: 227-243.

Azim, M. E., M. C. J. Verdegem, M. M. Rahman, M. A. Wahab, A. A. Van Dam, and M. C. M. Beveridge 2002a. Evaluation of polyculture of Indian major carps in periphyton-based ponds. Aquaculture 213: 131-149.

CFPCC. 2019. Annual report. Central Fisheries Promotion and Conservation Centre. Balaju, Kathmandu.

FAO. 2017. Nutrition-sensitive agriculture and food systems in practice Options for intervention. Rome, Italy.

Gangadhar, B., and P. Keshavanath 2012. Growth performance of rohu, Labeo rohita (Ham.) in tanks provided with different levels of sugarcane bagasse as periphyton substrate." Indian J. Fish 59: 77-82.

Gupta, M. C., and S. Rai 2011. Effect of Dedhuwa (Esomus danricus), Mara (Amblypharyngodon mola) and Pothi (Puntius sophore) on carp-prawn production in Chitwan, Nepal. Nepalese Journal of Aquaculture and Fisheries, 1: 73-84.

Gupta, M. C., and S. Rai 2014. Effect of Dedhuwa (Esomus danricus), Mara (Amblypharyngodon mola) and Pothi (Puntius sophore) on carp-prawn production in Chitwan, Nepal. Nepalese Journal of Aquaculture and Fisheries, 1: 73-84.

Increasing carp and small indigenous species production in semi-intensive ponds through enhancing periphyton growth. Report of the Special Session on Advancing Integrated Agriculture Aquaculture through Agroecology, Montpellier, France, 25 August 2018. FAO Fisheries and Aquaculture Report No. 1286. Rome.

Jha, S., S. Rai, M.K. Shrestha, J. S. Diana, R. B. Mandal, and H. Egna 2018. Production of periphyton to enhance yield in polyculture ponds with carps and small indigenous species." Aquaculture Reports 9: 74-81.
Milstein, A. 1992. Ecological aspects of fish species interactions in polyculture ponds. Hydrobiologia 231: 177-186.

Milstein, A., A. Naor, A. Barki, and S. Harpaz 2013. Utilization of periphytic natural food as partial replacement of commercial food in organic tilapia culture-an overview. Transylvanian Review of Systematical and Ecological Research 15(1): 49-60.

MoHP. 2006. Nepal Demographic and Health Survey 2006. Ministry of Health and Population, New Era, and Macro International Inc., Kathmandu.

Mridula, R. M., J. K. Manissary, P. Keshavnath, K. M. Shankar, M. C. Nandesha, and K.M. Rajesh 2003. Water quality, biofilm production and growth of fringe-lipped carp (Labeo fimbriatus) in tanks provided with two solid substrates. Aquaculture, 87: 263-267.

Mridula, R.M., J. K. Manissary, P. Keshavnath, K. M. Shankar, M. C. Nandesha, and K.M. Rajesh 2005. Effects of paddy straw and sugarcane bagasse on water quality, bacterial biofilm production and growth and survival of rohu, Labeo rohita (Hamilton). Aquaculture Research, 36: 635-642.

Rai, S., and Y.Yi 2012. Nibbling frequency of carps in fed and non-fed periphyton-based aquaculture system. The Israeli Journal of Aquaculture 64: 818-822.

Rai, S., K, Gharti, M. K. Shrestha, R. Ranjan, J. Diana, and H. Egna 2018. Potential substrates for periphyton enhancement in CarpSIS polyculture. Our Nature 16(1): 8-16.

Rai, S., K, Gharti, M. K. Shrestha, S. Jha, R. Poudel, R. Ranjan, J. Diana, and H. Egna 2019.

Rai, S., S. H. Thilsted, M. K. Shrestha, M. A. Wahab, and K. Gharti 2012. Improvement of women's livelihoods, income and nutrition through carp-sis-prawn polyculture in Terai, Nepal." Asian Fisheries Science Special Issue 25: 217-225.

Rai, S., S. H. Thilsted, M. K. Shrestha, M. A. Wahab, and M. C. Gupta 2014. Carp-SIS polyculture: A new intervention to improve women's livelihoods, income and nutrition in Terai, Nepal. Asian Fisheries Science Special Issue (Gender in Aquaculture and Fisheries: Navigating Change), 27S: 165174.

Rai, S., Y. Yi, M. A. Wahab, A. N. Bart, and J. S. Diana 2008. Comparison of rice straw 


\section{Our Nature | December 2020 | 18 (1): 38-48}

and bamboo stick substrates in periphytonbased carp polyculture systems. Aquaculture Research, 39(5): 464-473.

Rai, S., Y. Yi, Md A. Wahab, A. N. Bart, and J. S. Diana 2010. Comparison of the growth and production of carps in polyculture ponds with supplemental feed using rice straw and kanchi as substrates. Our Nature 8(1): 92-105.

Ramesh, M. R., K.M. Shankar, C. V. Mohan, and T. J. Varghese 1999. Comparison of three plant substrates for enhancing carp growth through bacterial biofilm. Aquaculture Engineering 19: 119-131.

Roos, N., M. A.Wahab, C. Chamman, and S. H. Thilsted 2006. Understanding the links between Agriculture and Health. 2020 vision for Food, Agriculture and Environment. International Food Policy Research. 2033 K Street, N. W. Washington, D. C. 20006-1002. USA Focus: 44-49.

Roos, N., Md A. Wahab, M. A. R. Hossain, and S. H. Thilsted 2007. Linking human nutrition and fisheries: Incorporating micronutrient-dense, small indigenous fish species in carp polyculture production in Bangladesh. Food and Nutrition Bullet-in 28(2): 280-S293.

Saikia, S. K., and D. N. Das 2009. Potentiality of periphyton-based aquaculture technology in rice-fish environment. Journal of Scientific Research 1(3): 624-634.
Shang, Y. C. 1990. Aquaculture economic analysis: an introduction. World Aquaculture Society. Baton Rouge, LA.

Shrestha, T. K. 2008. Ichthyology of Nepal, A study of Fishes of the Himalayan Waters. Himalayan Ecosphere, Kathmandu, Nepal.

Thilsted, S. H., N. Roos, and N. Hasan 1997. The role of small indigenous fish species in food and nutrition security in Bangladesh. The ICLARM Quarterly, July-Dec, 13.

UNICEF, 2012. A Milestone Plan Launched to Improve Maternal and Child Nutrition in Nepal. http://www.unicef.org/Nepal/5476_7891.ht m. (Accessed 8.06.2020).

Verdegem, M. C. J., and M. E. Azim 2001. Periphyton-based aquaculture: a novel fish culture technology. Research for sustainable development bulletin, 4, 37-39.

Wahab, M. A., M. A. Mannan, M. A. Huda, M. E Azim, A. G. Tollervey, and M. C. M. Beveridge 1999. Effects of periphyton grown on bamboo substrates on growth and production of Indian major carp, Rohu (Labeo rohita Ham.). Bangladesh Journal of Fish Research 3(1): 1-10.

Yadav, S.K., and S. Rai 2011. Production potential of carp-SIS-prawn polyculture in Chitwan, Nepal. Nepalese Journal of Aquaculture and Fisheries 1: 59-72.

Yadav, S.K., and S. Rai 2014. Production potential of carp-SIS-prawn polyculture in Chitwan, Nepal. 